\title{
General holographic superconductor models with Gauss-Bonnet corrections
}

\author{
Qiyuan $\operatorname{Pan}^{1,2,3}$, Bin Wang ${ }^{1}$ \\ 1 Department of Physics, Shanghai Jiao Tong University, Shanghai 200240, China \\ 2 Department of Physics, Fudan University, Shanghai 200433, China and \\ 3 Institute of Physics and Department of Physics, \\ Hunan Normal University, Changsha, Hunan 410081, China
}

\begin{abstract}
We study general models for holographic superconductors in Einstein-Gauss-Bonnet gravity. We find that different values of Gauss-Bonnet correction term and model parameters can determine the order of phase transitions and critical exponents of second-order phase transitions. Moreover we find that the size and strength of the conductivity coherence peak can be controlled. The ratios $\omega_{g} / T c$ for various model parameters have also been examined.

PACS numbers: $11.25 . \mathrm{Tq}, 04.70 . \mathrm{Bw}, 74.20 .-\mathrm{z}$
\end{abstract}




\section{INTRODUCTION}

The anti-de Sitter/conformal field theories (AdS/CFT) correspondence [1, 2] has provided a theoretical framework to describe the strongly coupled conformal field theories through a weakly coupled dual gravitational description. Recently stimulated from this correspondence, a remarkable connection between the condensed matter and the gravitational physics has been discovered, for reviews see [3-5]. It was suggested that the spontaneous $U(1)$ symmetry breaking by bulk black holes can be used to construct gravitational duals of the transition from normal state to superconducting state in the boundary theory $[6]$. One can look at a $(2+1)$-dimensional superconductor and see its striking image involving a charged black hole with non-trivial "hair" in (3+1)-dimensions [7]. This investigation was further carried out beyond the probe limit by considering the back-reaction of the field on the spacetime [8]. Gravity models with the property of holographic superconductor have attracted considerable interest for their potential applications to the condensed matter

physics, see for example $9-27]$. At the moment when the condensation occurs in the boundary CFT and in the gravitational counterpart a non-trivial hair for the black hole is triggered, there appears a phase transition. It was argued that this phase transition belongs to the second order [7]. The phenomenological signature of this phase transition was recently disclosed in the perturbation around such AdS black holes [28, 29].

Franco et al. recently introduced a generalization of the basic holographic superconductor model in which the spontaneous breaking of a global U(1) symmetry occurs via the Stückelberg mechanism [30]. They found that a generalized Stückelberg mechanism of symmetry breaking allows for a description of a wider class of phase transitions. This framework allows tuning the order of the phase transition which can accommodate the first order phase transition to occur, and for the second order phase transition it allows tuning the values of critical exponents [31]. An interesting extension was done in [32] by constructing general models for holographic superconductivity. It was found that except some universal model independent features, some important aspects of the quantum critical behavior strongly depend on the choice of couplings, such as the order of the phase transition and critical exponents of second-order phase transitions. In addition to the numerical investigation, analytical understanding on the phase transition of holographic superconductor was also provided in [33].

It is of great interest to generalize the investigation on the phase transition in the holographic superconductor to the Einstein-Gauss-Bonnet gravity. It was observed in recent works [19 21, 34] that the Gauss-Bonnet 
coupling affects the condensation and the higher curvature correction makes condensation harder to form. Further the high curvature correction also causes the behavior of the claimed universal ratio $\omega / T_{c} \approx 8$ unstable. In this work we are going to examine the effect of the Gauss-Bonnet correction on the order of the phase transition and critical exponents. We will also study the conductivity in a certain range of parameters and disclose the influence given by the Gauss-Bonnet coupling.

The plan of the work is the following. In Sec. II, we will study the phase transitions of a general class of the holographic superconductor models via the Stückelberg mechanism in the Gauss-Bonnet AdS black hole background. In Sec. III we will explore the effects of the Gauss-Bonnet correction terms and other model parameters on the conductivity. We will conclude in the last section of our main results.

\section{GENERAL SUPERCONDUCTING MODELS IN GAUSS-BONNET ADS BLACK HOLE}

We will consider the background solution of a neutral black hole in $d$ dimensional Einstein-Gauss-Bonnet gravity [35, 36]

$$
d s^{2}=-f(r) d t^{2}+\frac{d r^{2}}{f(r)}+r^{2} d x_{i} d x^{i}
$$

with

$$
f(r)=\frac{r^{2}}{2 \alpha}\left[1-\sqrt{1-\frac{4 \alpha}{L^{2}}\left(1-\frac{M L^{2}}{r^{d-1}}\right)}\right],
$$

where $M$ is a constant of integration relating to the black hole horizon by $r_{+}=\left(M L^{2}\right)^{1 /(d-1)}, \alpha$ is the GaussBonnet coupling constant and $L$ is the AdS radius. The Gauss-Bonnet correction $\alpha$ has an upper bound called the Chern-Simons limit $\alpha=L^{2} / 4$, and a lower bound determined by the causality [37, 38]. In the limit $\alpha \rightarrow 0$, (1) goes back to the Schwarzschild AdS black hole.

Consider a $U(1)$ gauge field and the scalar field coupled via a generalized Stückelberg Lagrangian 31]

$$
S=\int d^{d} x \sqrt{-g}\left[-\frac{1}{4} F_{\mu \nu} F^{\mu \nu}-\frac{1}{2} \partial_{\mu} \tilde{\psi} \partial^{\mu} \tilde{\psi}-\frac{1}{2} m^{2} \tilde{\psi}^{2}-\frac{1}{2}|\mathfrak{F}(\tilde{\psi})|\left(\partial_{\mu} p-A_{\mu}\right)\left(\partial^{\mu} p-A^{\mu}\right)\right],
$$

with gauge symmetry $A_{\mu} \rightarrow A_{\mu}+\partial_{\mu} \Lambda$ and $p \rightarrow p+\Lambda$. $\mathfrak{F}$ is a general function of $\tilde{\psi}$ which has the following form

$$
\mathfrak{F}(\tilde{\psi})=\tilde{\psi}^{2}+c_{\gamma} \tilde{\psi}^{\gamma}+c_{4} \tilde{\psi}^{4}
$$

with the model parameters $c_{\gamma}, \gamma$ and $c_{4}$. When $c_{\gamma}$ and $c_{4}$ are zero, it reduces to the model considered in [19, 20]. 
Using the gauge freedom to fix $p=0$ and taking the ansatz $\psi \equiv \tilde{\psi}, A_{t}=\phi$ where $\psi, \phi$ are both real functions of $r$ only, we can obtain the equations of motion

$$
\begin{aligned}
& \psi^{\prime \prime}+\left(\frac{f^{\prime}}{f}+\frac{d-2}{r}\right) \psi^{\prime}+\frac{\phi^{2}}{2 f^{2}} \mathfrak{F}^{\prime}(\psi)-\frac{m^{2}}{f} \psi=0, \\
& \phi^{\prime \prime}+\frac{d-2}{r} \phi^{\prime}-\frac{\mathfrak{F}(\psi)}{f} \phi=0 .
\end{aligned}
$$

These two equations can be solved numerically by doing integration from the horizon out to the infinity. At the asymptotic AdS boundary $(r \rightarrow \infty)$, the solutions behave like

$$
\psi=\frac{\psi_{-}}{r^{\lambda_{-}}}+\frac{\psi_{+}}{r^{\lambda_{+}}}, \quad \phi=\mu-\frac{\rho}{r^{d-3}},
$$

with

$$
\lambda_{ \pm}=\frac{1}{2}\left[(d-1) \pm \sqrt{(d-1)^{2}+4 m^{2} L_{\text {eff }}^{2}}\right]
$$

where $L_{\text {eff }}^{2}=2 \alpha /\left(1-\sqrt{1-4 \alpha / L^{2}}\right)$ is the effective asymptotic AdS scale [19, 20], $\mu$ and $\rho$ are interpreted as the chemical potential and charge density in the dual field theory respectively. Notice that both of the falloffs are normalizable for $\psi$, so one can impose boundary condition that either $\psi_{+}$or $\psi_{-}$vanishes [7, 8]. For simplicity, we will take $\psi_{-}=0$. Moreover, we will set $d=5$ and $m^{2} L^{2}=-3$ for concreteness. As a matter of fact, the other choices will not qualitatively modify our results. Thus, the scalar condensate is now described by the operator $\left\langle\mathcal{O}_{+}\right\rangle=\psi_{+}$and we will discuss the condensate $\left\langle\mathcal{O}_{+}\right\rangle$for fixed charge density.

We will investigate how the phase transition depends on the coefficients $c_{\gamma}$ and $c_{4}$. Due to the special interest in the case $\mathfrak{F}(\psi)=\psi^{2}+c_{4} \psi^{4}$ [17, 18], in (4) we are going to set $c_{\gamma}=0$ for the moment and pay more attention on the influence of $c_{4}$ on the phase transition. Solving the equations of motion numerically, in Fig 1 we plot the condensate around the critical region for chosen values of $c_{4}$ and different Gauss-Bonnet constants. For $0 \leq c_{4}<0.3$, the transition is second order and the condensate approaches zero as $\left\langle\mathcal{O}_{+}\right\rangle \sim\left(T_{c}-T\right)^{\beta}$, with mean field critical exponent $\beta=1 / 2$ for all values of $\alpha$. For $c_{4} \geq 1$, we observe that $\left\langle\mathcal{O}_{+}\right\rangle$becomes multivalued near the critical temperature and the condensate does not drop to zero continuously at the critical temperature. This behavior keeps for all values of $\alpha$. The analogous phenomenon holds as well when we consider the $\left\langle\mathcal{O}_{-}\right\rangle$condensate but with different $c_{4}$ range. In [31], for the case without Gauss-Bonnet constant, it was argued that the behavior for $c_{4} \geq 1$ indicates that the phase transition changes from the second order to the first order at $c_{4}=1$. Here we find that the Gauss-Bonnet constant does not alter the result when $c_{4} \geq 1$. Choosing $c_{4} \in[0.3,1.0]$, we observe in Fig. 1 that for fixed $c_{4}$, the transition point of the

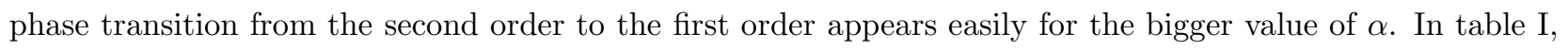


we list the critical value of $\alpha_{c}$ separating the second order and the first order phase transitions for selected $c_{4}$ within the range $[0.3,1.0]$. Since we concentrate on the five-dimensional spacetime, the separation point between the second and the first order phase transitions does not coincide with that in four dimensions, for example $\left(c_{4}=0.9, \alpha_{c}=0\right)$ here and $\left(c_{4}=1, \alpha=0\right)$ in 31$]$. With the increase of $c_{4}$, we see that $\alpha_{c}$ becomes smaller. Thus we find that when $c_{4} \in[0.3,1.0]$, not only $c_{4}$ but also the Gauss-Bonnet constant can tune the order of the phase transition. The Gauss-Bonnet constant provides richer physics in the phase transition.

TABLE I: The critical value of $\alpha_{c}$ which can separate the first- and second-order behavior for different $\mathfrak{F}(\psi)=\psi^{2}+c_{4} \psi^{4}$.

\begin{tabular}{|c|c|c|c|c|c|}
\hline$c_{4}$ & 0.3 & 0.5 & 0.7 & 0.9 & 1.0 \\
\hline$\alpha_{c}$ & 0.25 & 0.2 & 0.1 & 0.0 & -0.1 \\
\hline
\end{tabular}
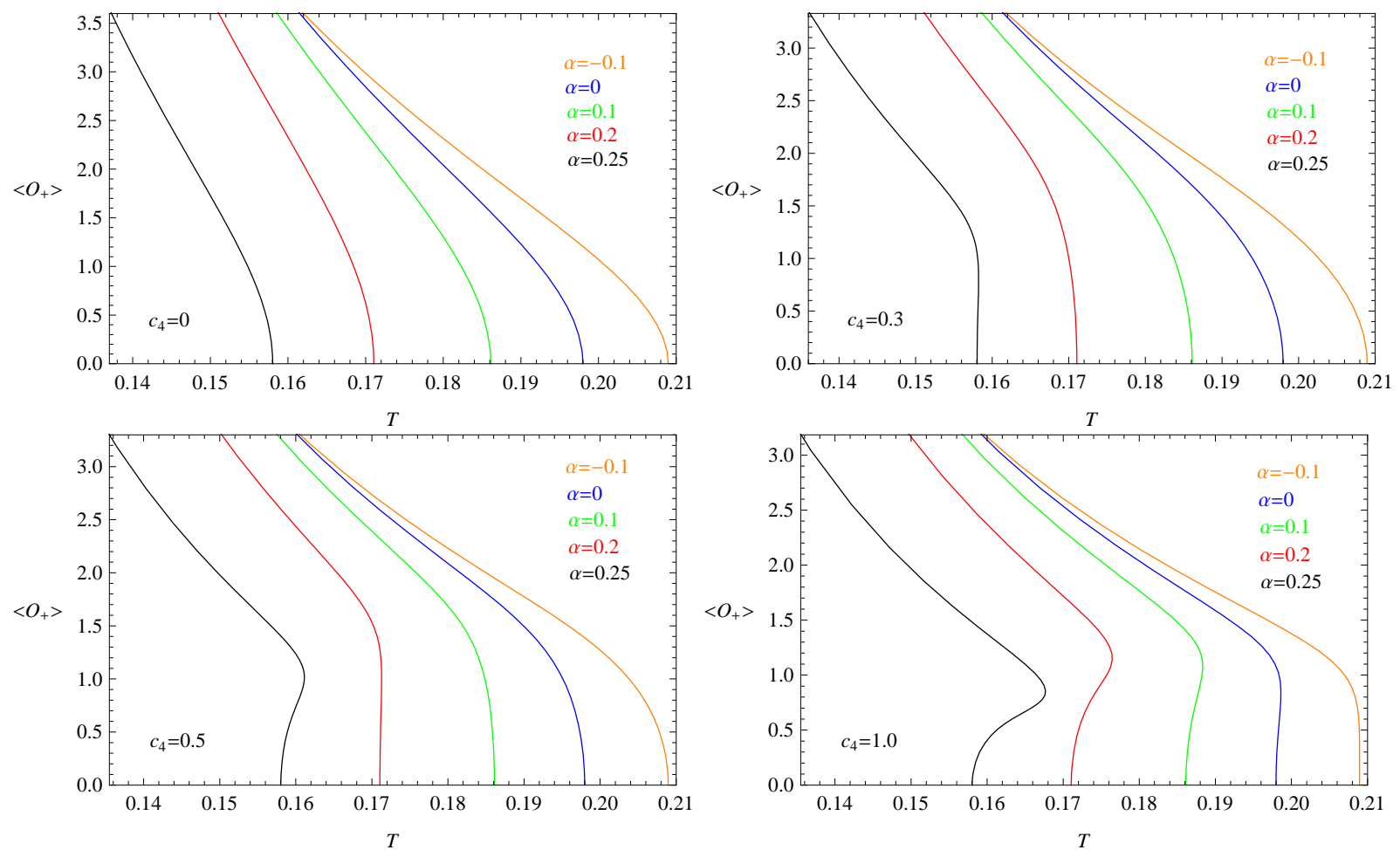

FIG. 1: (Color online) The condensate $\left\langle\mathcal{O}_{+}\right\rangle$as a function of temperature with fixed values $c_{4}\left(c_{\gamma}=0\right)$ for different values of $\alpha$, which shows that a different value of $\alpha$ can separate the first- and second-order behavior. The five lines in each panel from left to right correspond to decreasing $\alpha$, i.e., 0.25 (black), 0.2 (red), 0.1 (green), 0 (blue) and -0.1 (orange).

We also have the interest to see the influence of $c_{\gamma}$ on the phase transition. We will concentrate on $\gamma$ in the range $3 \leq \gamma \leq 4$. At this moment we set $c_{4}=0$. In Fig. 2 we exhibit the condensate of $<\mathcal{O}_{+}>$for selected values of $c_{\gamma}, \alpha$ and changing $\gamma$. We see that for fixed small values of $\alpha$ and $c_{\gamma}$, it is more possible to observe the appearance of the first order phase transition when $\gamma$ is smaller. The $\alpha$ influence on the order of the phase transition is also exhibited. Bigger $\alpha$ will cause the first order phase transition to appear easier for 
fixed $c_{\gamma}$ and $\gamma$. This is consistent with the case for nonzero $c_{4}$ but zero $c_{\gamma}$. The effect of $c_{\gamma}$ is similar to the Gauss-Bonnet constant, bigger $c_{\gamma}$ brings the first order phase transition easier for fixed $\gamma$ and $\alpha$.
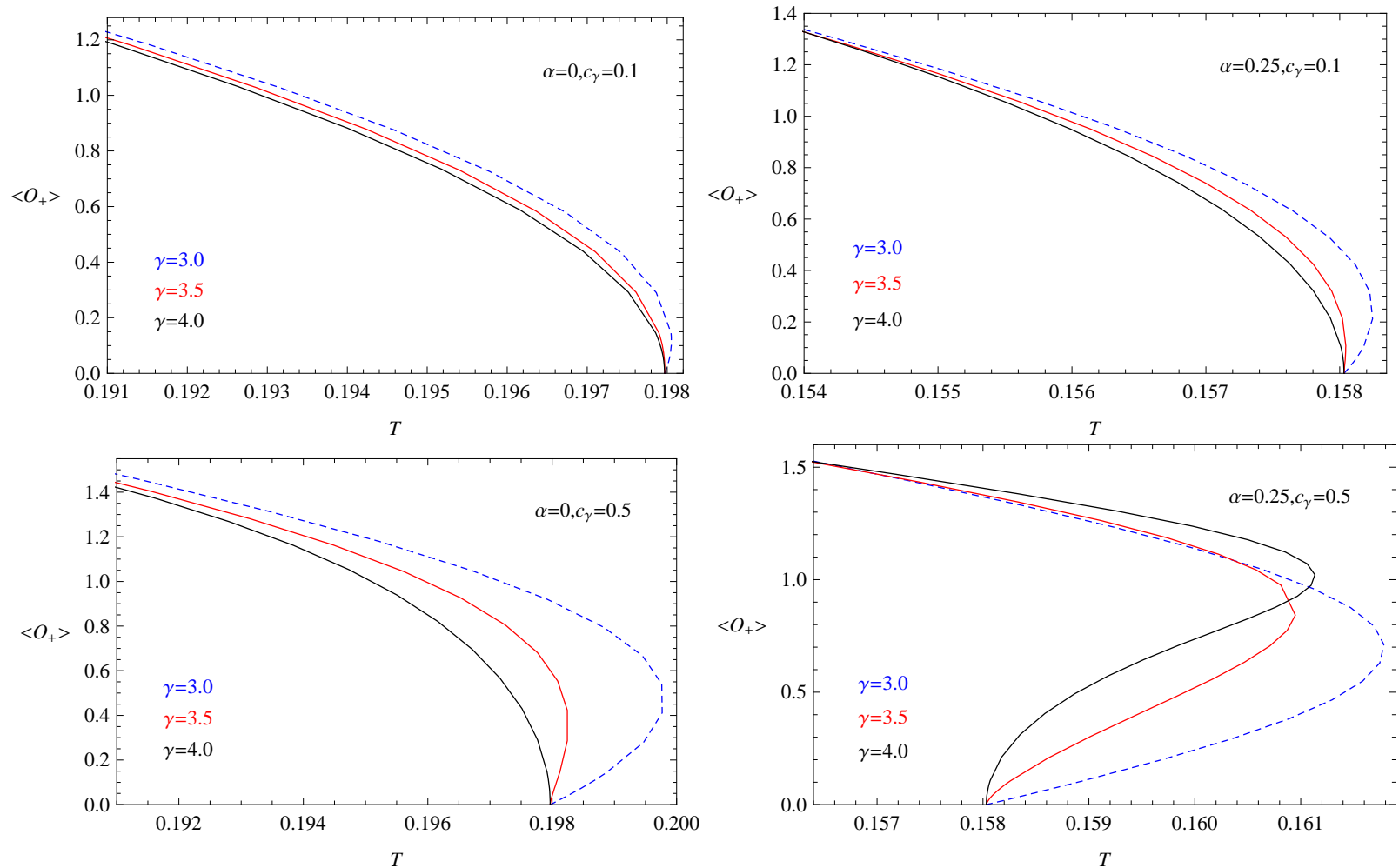

FIG. 2: (Color online) The condensate $\left\langle\mathcal{O}_{+}\right\rangle$as a function of temperature with fixed values $c_{\gamma}$ and $\alpha$ for different values of $\gamma$, which shows that the type of phase transition depends on the Gauss-Bonnet correction term $\alpha$, model parameters $\gamma$ and $c_{\gamma}$. The three lines in each panel from left to right correspond to decreasing $\gamma$, i.e., 4.0 (black), 3.5 (red) and 3.0 (blue and dashed).

In addition to showing that the gravity duals can lead to both first and second order phase transitions, it is also of great interest to examine in the second order phase transition whether different choices of $c_{\gamma}, c_{4}, \gamma, \alpha$ result in different critical exponents from the prediction of the mean field. When $c_{\gamma}=0$ and $c_{4}=0$, the critical exponent was shown in agreement with the mean field value $\beta=1 / 2$ in the Einstein-Gauss-Bonnet gravity [19, 20]. An interesting behavior arises for $c_{\gamma}<0$. In Fig. 3, for $c_{\gamma}=-1$ and $c_{4}=1 / 2$, we present the condensate $\left\langle\mathcal{O}_{+}>\right.$as a function of $1-T / T_{c}$ in logarithmic scale with different values of $\alpha$ for choosing $\gamma=3.0,3.25,3.5$ and 4.0 respectively. Three lines in each panel from the bottom to the top correspond to $\alpha=0.25,-0.1$ and 0.1 . We see that the slope is independent of the Gauss-Bonnet correction term $\alpha$ but sensitive to the model parameter $\gamma$. Further analysis shows that, near the critical temperature $T_{c}$, the critical exponent $\beta \simeq 1.00$ for $\gamma=3.0, \beta \simeq 0.79$ for $\gamma=3.25, \beta \simeq 0.67$ for $\gamma=3.5$ and $\beta \simeq 0.50$ for $\gamma=4.0$, which is 
independent of $\alpha$ ! The relation between the critical exponent $\beta$ and the parameter $\gamma$ can be expressed as

$$
\beta \cong \frac{1}{\gamma-2}
$$

This behavior is consistent with that seen for the $\mathrm{AdS}_{4}$ black hole with the scalar mass $m^{2} L^{2}=-2$ [31, 32], which tells us that the critical exponent $\beta$ depends only on the model parameter $\gamma$ but is independent of the scalar mass and the background spacetimes.
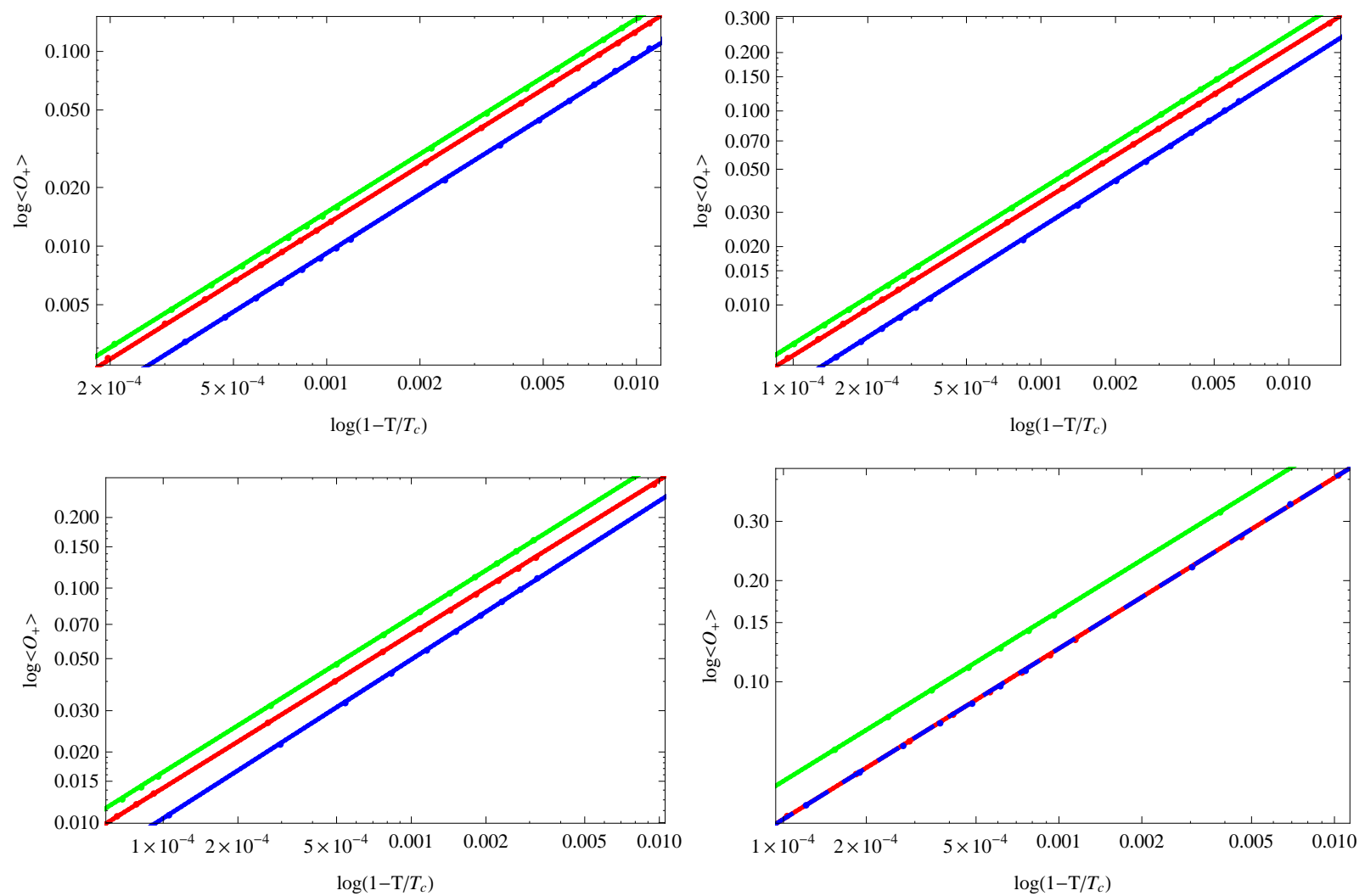

FIG. 3: (color online) The condensate $\left\langle\mathcal{O}_{+}\right\rangle$vs $1-T / T_{c}$ in logarithmic scale with different values of $\alpha$ for $\gamma=3.0$ (top and left), 3.25 (top and right), 3.5 (bottom and left) and 4.0 (bottom and right). The three lines in each panel from bottom to top correspond to $\alpha=0.25$ (blue), -0.1 (red) and 0.1 (green). These panels show that the slope is independent of $\alpha$ but sensitive to $\gamma$.

In Fig. 4 we present the condensate as a function of temperature for $c_{\gamma}=-1$ and $c_{4}=1 / 2$. It exhibits that the critical temperature $T_{c}$ is independent of the model parameter $\gamma$ but depends on the Gauss-Bonnet correction term $\alpha$, i.e., $T_{c}=0.209$ for $\alpha=-0.1, T_{c}=0.198$ for $\alpha=0, T_{c}=0.186$ for $\alpha=0.1$ and $T_{c}=0.158$ for $\alpha=0.25$, which shows that the positive Gauss-Bonnet correction will suppress the condensation but the negative one will enhance it [21]. In fact, we note that the critical temperature $T_{c}$ is not sensitive to the coefficients $c_{\gamma}$ and $c_{4}$ in the general function $\mathfrak{F}(\psi)$ from Figs. 1, 2, and 4 for the phase transition of the second order. Thus, we point out that the critical temperature $T_{c}$ depends on the background spacetimes but not on 
the model of $\mathfrak{F}$.
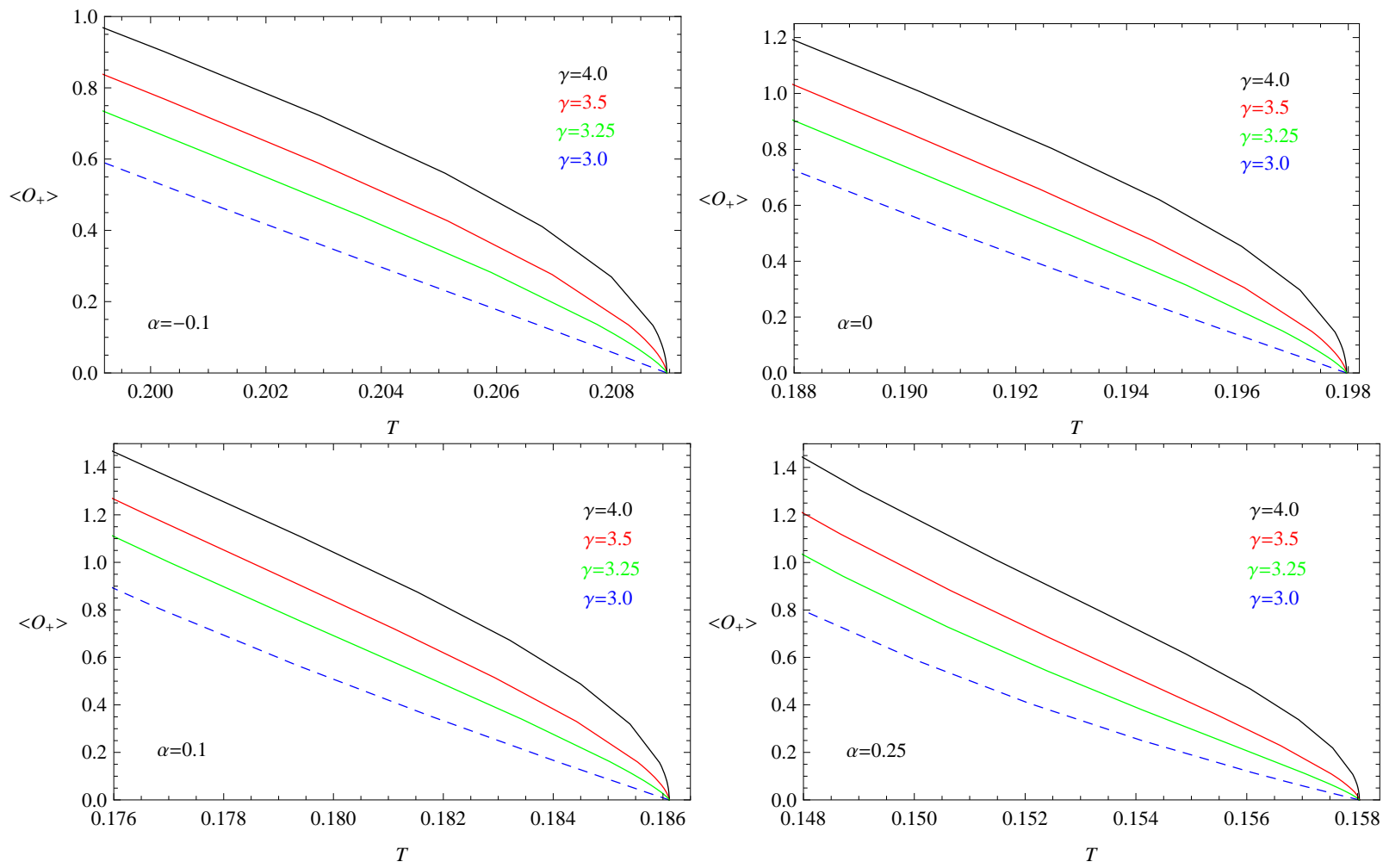

FIG. 4: (color online) The condensate $\left\langle\mathcal{O}_{+}\right\rangle$as a function of temperature with fixed values $\alpha$ for different model parameters $\gamma$. The four lines in each panel from bottom to top correspond to increasing $\gamma$, i.e., 3.0 (blue and dashed), 3.25 (green), 3.5 (red) and 4.0 (black). These panels show that the critical temperature $T_{c}$ is independent of $\gamma$ but depends on $\alpha$.

\section{CONDUCTIVITY}

Now we jump to investigate the influence of the Gauss-Bonnet correction term $\alpha$, model parameters $\gamma, c_{\gamma}$ and $c_{4}$ in $\mathfrak{F}(\psi)$ on the conductivity.

In order to calculate the conductivity, we consider the perturbed Maxwell field $\delta A_{x}=A_{x}(r) e^{-i \omega t} d x$. The equation of motion for $\delta A_{x}$ reads

$$
A_{x}^{\prime \prime}+\left(\frac{f^{\prime}}{f}+\frac{d-4}{r}\right) A_{x}^{\prime}+\left[\frac{\omega^{2}}{f^{2}}-\frac{\mathfrak{F}(\psi)}{f}\right] A_{x}=0
$$

We still restrict our study to $d=5$ in order to avoid the complicated behavior in the gauge field falloff in dimensions higher than five. We solve the above equation by imposing the ingoing boundary condition

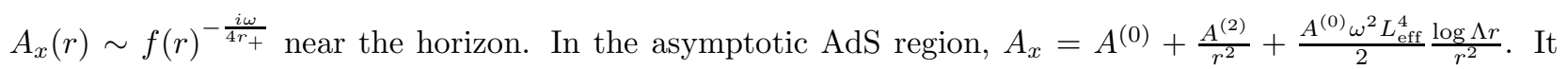
should be noted that the appearance of the arbitrary integration constant, $\Lambda$, leads to a logarithmic divergence in the retarded Green's function $G^{R}$ which gives the conductivity $\sigma$. In order to remove the divergent term 
$\log \Lambda r$, we add a boundary counter term in the gravity action as suggested in [39], which can specify the renormalization scale when regulating the action [9]. With this appropriate boundary counter term to cancel the logarithmic divergence, we can express the conductivity as 9 , 19, 20]

$$
\sigma=\frac{2 A^{(2)}}{i \omega A^{(0)}}+\frac{i \omega}{2}
$$

For the general forms of function $\mathfrak{F}(\psi)=\psi^{2}+c_{\gamma} \psi^{\gamma}$ (setting $c_{4}=0$ for clarity), one can obtain the conductivity by solving the Maxwell equation numerically. We will focus on the case for the fixed scalar mass $m^{2} L^{2}=-3$ in our discussion.
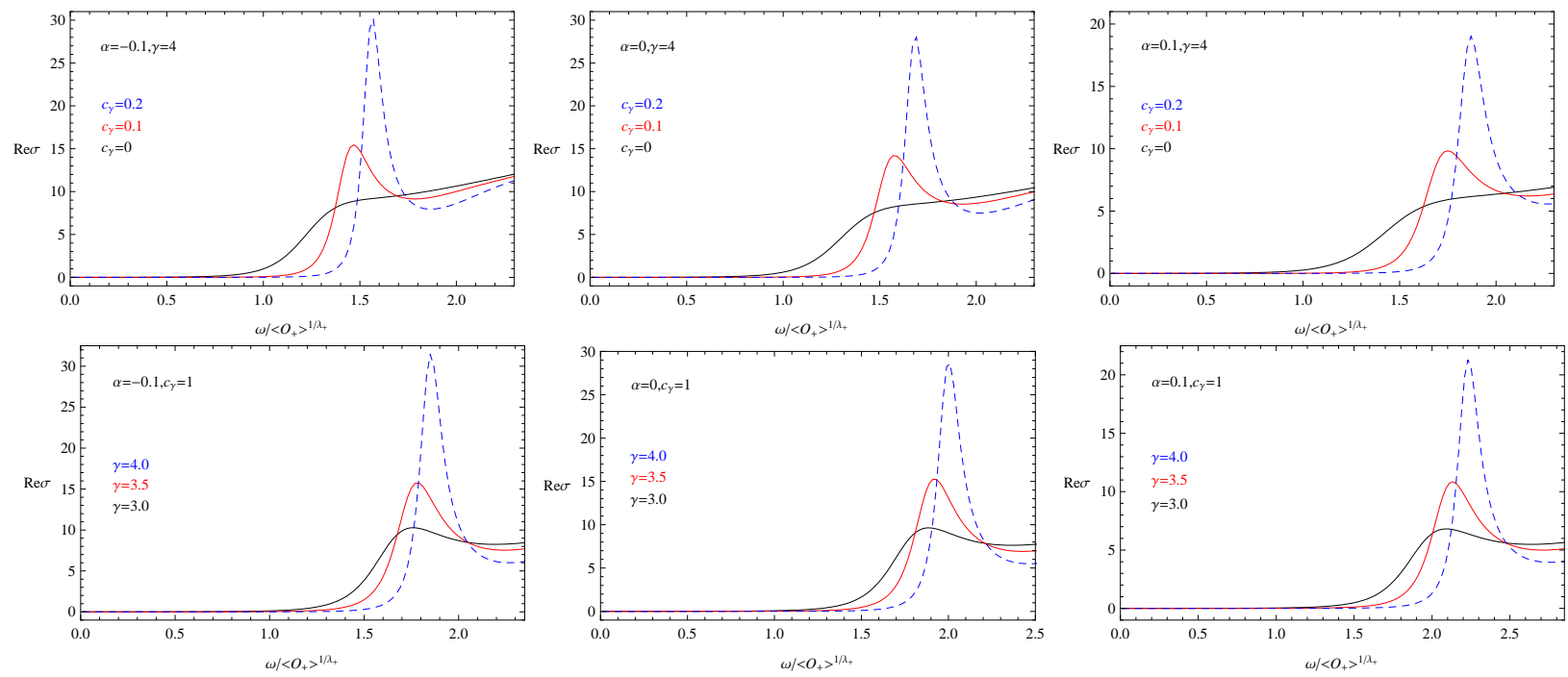

FIG. 5: (color online) The real part of the conductivity for $(3+1)$-dimensional Gauss-Bonnet superconductors with different values of $\gamma, c_{\gamma}$ and $\alpha$.

In the top-three panels in Fig. [5 we have plotted $\operatorname{Re} \sigma(\omega)$ by fixing $\gamma=4$ for different values of $c_{\gamma}$ and $\alpha$. It clearly shows that for fixed $\alpha$ the coherence peak gradually becomes stronger and narrower with the increase of $c_{\gamma}$. This is consistent with the result of $c_{4}$ observed in [31], which indicates that $c_{\gamma}$ controls the magnitude of the fluctuations of the condensate. For bigger $c_{\gamma}$ we have already seen that the fluctuations are strong enough to induce the first order phase transition. In the bottom-three panels in Fig. [5 we show the result by fixing $c_{\gamma}=1$ and varying $\gamma$ with selected $\alpha$ at $T / T_{c} \simeq 0.7$. We observe that with the increase of $\gamma$ the coherence peak increases for the same $\alpha$, which shows that the model parameter $\gamma$ also controls the magnitude of the fluctuations. From Fig. 5 we also learn that for the selected $c_{\gamma}$ and $\gamma$, the coherence peak becomes lower with the larger $\alpha$, which shows that the fluctuations in holographic superconductors will be suppressed by the higher order curvature corrections.

In [9] it was argued that there is a universal relation between the gap $\omega_{g}$ in the frequency dependent 
conductivity and the critical temperature $T_{c}: \omega_{g} / T_{c} \approx 8$ respected to a good approximation by all cases considered. However this claimed universal relation was challenged when the higher curvature corrections are taken into account [19, 20]. The available discussions were in the model when $\mathfrak{F}(\psi)=\psi^{2}$. In the general $\mathfrak{F}$ form in our work, we will show that the model parameters $\gamma, c_{\gamma}$ also modify the claimed universal relation even when $\alpha=0$. In Fig. [6 we plot the conductivity at temperature $T / T_{c} \simeq 0.3$. The blue (bottom) line represents the real part of the conductivity and the red (top) line is the imaginary part. We can easily find a gap in the conductivity with the gap frequency $\omega_{g}$ changes with the values of Gauss-Bonnet correction term $\alpha$, model parameters $\gamma$ and $c_{\gamma}$. Fixing $\gamma$ and $c_{\gamma}$, the gap frequency $\omega_{g}$ becomes larger for bigger $\alpha$, which agrees with the finding in [19, 20]. The gap frequency $\omega_{g}$ increases with $\gamma$ for fixed $c_{\gamma}, \alpha$ and grows with $c_{\gamma}$ for selected $\gamma, \alpha$. The deviation from $\omega_{g} / T_{c}=8$ becomes bigger with the increase of $\gamma$ and $c_{\gamma}$. This holds even when $\alpha=0$. This shows that not only the high curvature correction, but also the form of the scalar field $\mathfrak{F}$ will affect the so-called universal relation $\omega_{g} / T_{c} \approx 8[9]$.
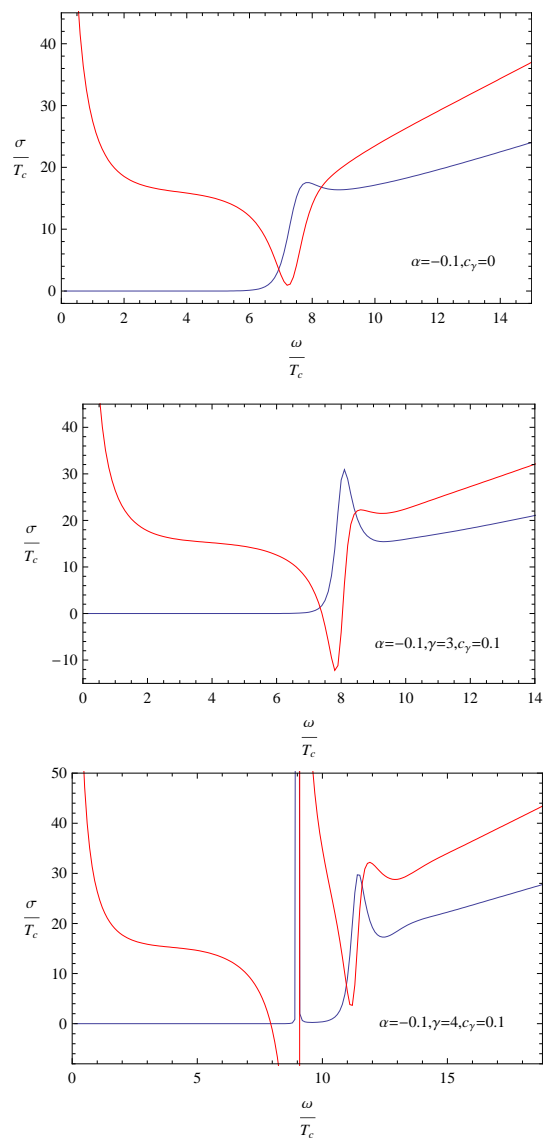
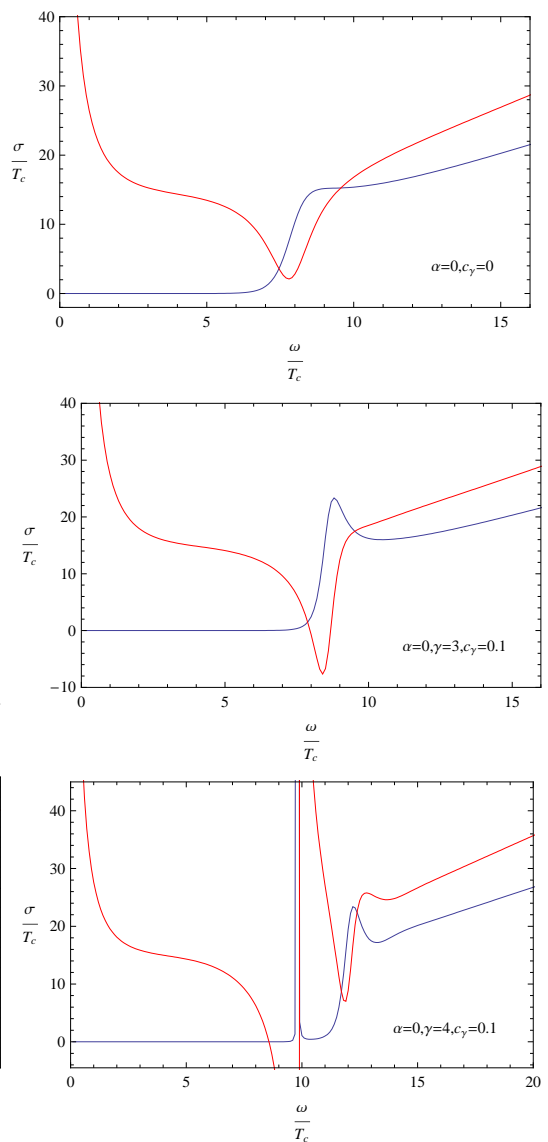
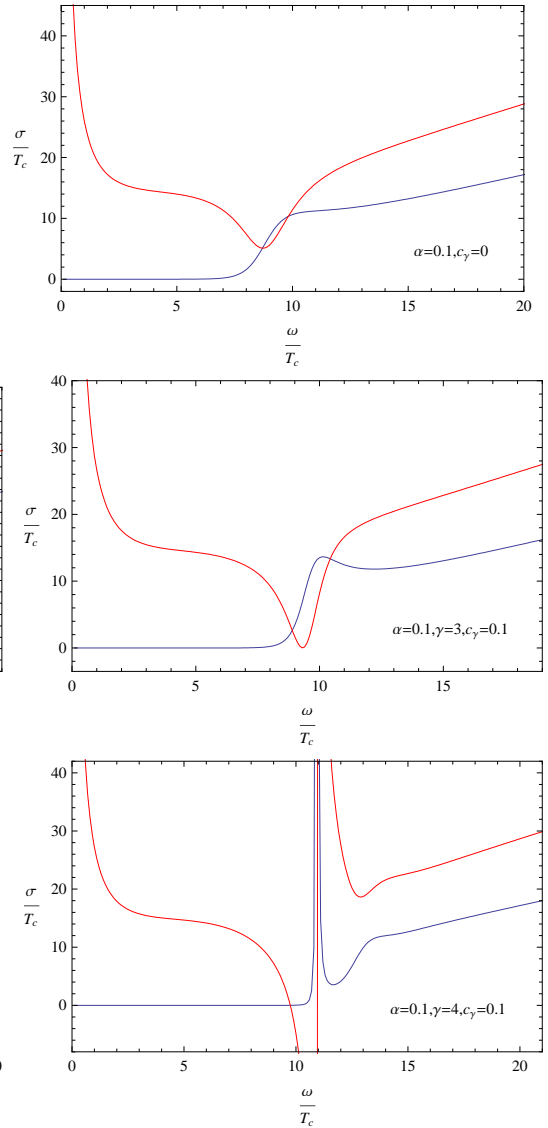

FIG. 6: (color online) Conductivity for $(3+1)$-dimensional Gauss-Bonnet superconductors with fixed values of $\alpha$ for different models with $\mathfrak{F}(\psi)=\psi^{2}+c_{\gamma} \psi^{\gamma}$. The blue (bottom) line and red (top) line represent the real part and imaginary part of the conductivity respectively. 


\section{CONCLUSIONS}

We have introduced a general class of gravity dual with Gauss-Bonnet corrections to describe both first and second order phase transitions at finite temperature in strongly interacting systems. In the probe limit, we found that besides the parameters which define $\mathfrak{F}$ can separate the first and second order phase transitions, different values of the Gauss-Bonnet correction bring richer physics and can also change the order of the phase transition in the generalized system. However for the second order phase transition, we observed that the shift of the critical exponents from that of the mean field result only appears for the parameters defining $\mathfrak{F}$ and is independent of the Gauss-Bonnet coupling. This is different from what we observed for the critical temperature $T_{c}$, which only depends on the Gauss-Bonnet constant while has nothing to do with other model parameters.

We also discussed the influences of the Gauss-Bonnet corrections and other model parameters on the conductivity. We found that the size and strength of the coherence peak can not only be controlled by the parameters that define $\mathfrak{F}$ as observed in [31], but also be influenced by the Gauss-Bonnet coupling. This shows that $\mathfrak{F}$ together with the high curvature correction controls the magnitude of the fluctuations in the system. Furthermore we examined the relation between the gap $\omega_{g}$ in the frequency dependent conductivity and the critical temperature $T_{c}$. We found that in addition to the high curvature influence on the ratio $\omega_{g} / T_{c}$ observed in [19, 20], the model parameters defining $\mathfrak{F}$ also give corrections to the so-called universal relation $\omega_{g} / T_{c} \approx 8$

\section{Acknowledgments}

This work was partially supported by the National Natural Science Foundation of China. Qiyuan Pan was also supported by the China Postdoctoral Science Foundation.

[1] J. Maldacena, Adv. Theor. Math. Phys. 2, 231 (1998); Int. J. Theor. Phys. 38, 1113 (1999).

[2] E. Witten, Adv. Theor. Math. Phys. 2, 253 (1998).

[3] S.A. Hartnoll, Class. Quant. Grav. 26, 224002 (2009).

[4] C.P. Herzog, J. Phys. A 42, 343001 (2009).

[5] G.T. Horowitz, arXiv: 1002.1722 [hep-th].

[6] S.S. Gubser, Phys. Rev. D 78, 065034 (2008).

[7] S.A. Hartnoll, C.P. Herzog, and G.T. Horowitz, Phys. Rev. Lett. 101, 031601 (2008).

[8] S.A. Hartnoll, C.P. Herzog, and G.T. Horowitz, J. High Energy Phys. 12, 015 (2008).

[9] G.T. Horowitz and M.M. Roberts, Phys. Rev. D 78, 126008 (2008).

[10] E. Nakano and W.Y. Wen, Phys. Rev. D 78, 046004 (2008).

[11] I. Amado, M. Kaminski, and K. Landsteiner, J. High Energy Phys. 05, 021 (2009).

[12] G. Koutsoumbas, E. Papantonopoulos, and G. Siopsis, J. High Energy Phys. 07, 026 (2009). 
[13] O.C. Umeh, J. High Energy Phys. 08, 062 (2009).

[14] K. Maeda, M. Natsuume, and T. Okamura, Phys. Rev. D 79, 126004 (2009).

[15] H.B. Zeng, Z.Y. Fan, and Z.Z. Ren, Phys. Rev. D 80, 066001 (2009).

[16] J. Sonner, Phys. Rev. D 80, 084031 (2009).

[17] S.S. Gubser, C.P. Herzog, S.S. Pufu, and T. Tesileanu, Phys. Rev. Lett. 103, 141601 (2009).

[18] J.P. Gauntlett, J. Sonner, and T. Wiseman, Phys. Rev. Lett. 103, 151601 (2009).

[19] R. Gregory, S. Kanno, and J. Soda, J. High Energy Phys. 10, 010 (2009).

[20] Q.Y. Pan, B. Wang, E. Papantonopoulos, J. Oliveria, and A.B. Pavan, Phys. Rev. D 81, 106007 (2010).

[21] X.H. Ge, B. Wang, S.F. Wu, and G.H. Yang, arXiv:1002.4901 [hep-th].

[22] R.G. Cai and H.Q. Zhang, Phys. Rev. D 81, 066003 (2010).

[23] J.L. Jing, L.C. Wang, and S.B. Chen, arXiv:1001.1472 [hep-th].

[24] S.B. Chen, L.C. Wang, C.K. Ding, and J.L. Jing, Nucl. Phys. B 836, 222 (2010).

[25] R.A. Konoplya and A. Zhidenko, Phys. Lett. B 686, 199 (2010).

[26] T. Nishioka, S. Ryu, and T. Takayanagi, J. High Energy Phys. 03, 131 (2010).

[27] G. Siopsis and J. Therrien, J. High Energy Phys. 05, 013 (2010).

[28] X. He, B. Wang, R.G. Cai, and C.Y. Lin, Phys. Lett. B 688, 230 (2010); arXiv:1002.2679 [hep-th].

[29] R.G. Cai, Z.Y. Nie, B. Wang, and H.Q. Zhang, arXiv:1005.1233 [gr-qc].

[30] S. Franco, A.M. Garcia-Garcia, and D. Rodriguez-Gomez, J. High Energy Phys. 04, 092 (2010).

[31] S. Franco, A.M. Garcia-Garcia, and D. Rodriguez-Gomez, Phys. Rev. D 81, 041901(R) (2010).

[32] F. Aprile and J.G. Russo, Phys. Rev. D 81, 026009 (2010).

[33] C.P. Herzog, Phys. Rev. D 81, 126009 (2010); arXiv:1003.3278 [hep-th].

[34] Y. Brihaye and B. Hartmann, Phys. Rev. D 81, 126008 (2010); arXiv:1003.5130 [hep-th].

[35] D.G. Boulware and S. Deser, Phys. Rev. Lett. 55, 2656 (1985).

[36] R.G. Cai, Phys. Rev. D 65, 084014 (2002).

[37] A. Buchel and R.C. Myers, J. High Energy Phys. 08, 016 (2009).

[38] X.O. Camanho and J.D. Edelstein, arXiv:0911.3160 [hep-th]; arXiv:0912.1944 [hep-th].

[39] M. Taylor, arXiv:hep-th/0002125 